\title{
Is the Atomic Second too short?
}

\author{
Ci-yuan $\mathrm{Liu}^{\star}$ \\ Shaanxi Observatory and National Observatories, Chinese Academy of Sciences, Lintong 710600, China \\ Received 8 January 2001 / Accepted 21 February 2001

\begin{abstract}
The definition of the length of the Atomic Second is based on measurements of the Earth's rotation made during the past 300 years. It has become too short because of the long-term deceleration of the Earth's rotation, making it necessary to add more and more leap seconds to UTC. By defining a longer Atomic Second and enlarging the limit of UTC from UT1, we would not need any leap second in the next dozens of years.
\end{abstract}

Key words. definition of second - atomic time - earth's rotation - UTC

In the past, the rotating Earth has been used as the standard clock. The length of day (Universal Time or UT) was the standard unit of time, and 1/86400 day was a standard second (UT second). Astronomers and geophysicists have shown that the Earth's rotation is far from stable and even. Its spectrum has many components with periods ranging from days to years. There are also irregular decade perturbation and long-term monotonic deceleration (Archinal 1992; Dickey 1994). A more stable unit of time (Ephemeris Time or ET) based on the revolution of the Earth around the Sun, was later defined. Because of the difficulty of observational determinations and other reasons, it was replaced by Atomic Time. The Atomic Second (SI second) is defined as the duration of 9192631770 periods of the radiation corresponding to the transition between two hyperfine levels of the ground state of the cesium-133 atom. This constant is the result of comparing the length of an ET second with atomic clock during 1954-1958. To keep in step with the Earth's rotation, UT (the natural day), Coordinated Universal Time (UTC) adopts the Atomic Second in a Universal Time frame. When the difference between UTC and UT (actually UT1) becomes large enough, a "leap second" is added to or subtracted from UTC to follow UT. According to the present regulation, a leap second is added or subtracted at the end of June 30 or December 31, so that the difference between UTC and UT1 is less than $0.9 \mathrm{~s}$. Through monitoring to the Earth's rotation by astronomical methods, when and what ( + or - ) leap second is needed is decided. Usually this information is announced 3-4 months earlier. UTC is now the standard civil time everywhere in the world (Seidelmann 1992).

The leap second, added or subtracted can effectively adjust for the fluctuations in the Earth's rotation.

* e-mail: liucy@ms.sxso.ac.cn
However, because of the long-term deceleration of the Earth rotation, the second in UT, 1/86400 part of a day, will be longer and longer, so leap seconds will have to be added more and more frequently. When as many as $3 \mathrm{~s}$ have to be added in one year, we will have to think of a new, longer definition of the Atomic Second because of the practical inconvenience. According to BIPM (Bureau International des Poids et Mesures) annual report (1997), in the last 27 years (1972.7-1999.1), 21 leap seconds have been added to UTC, or 7.8 for every 10 years.

Now the long-term deceleration of the Earth's rotation can be approximated as

$\Delta T=c t^{2}$

where, the clock error $\Delta T$ in seconds is the difference between AT (ET) and UT; $t$ is the number of century from 1800 AD. Ancient astronomical records have been used to determine the constant $c$. Liu (1994) and Pang (1995) indicated that $c=30 \mathrm{sec} c \mathrm{y}^{-2}$ is reasonable, while Stephenson (1995) prefer $c=31$. The little difference does not affect our discussion here. Neglecting the decade fluctuations, the long-term deceleration would require the addition of more and more leap seconds $(L)$ in UTC as shown in Table 1 ( $\operatorname{supposing} c=30$ ).

Here, $E$ stands for the number of years from an epoch when seconds of AT equal those of UT; $\Delta T$ is in second and $L$ denotes how many leap seconds have to be added every ten years.

From Table 1 we see that $\Delta T$ increases parabolically, while $L$ linearly. We can bear a difference between even time AT and civil time UTC, but it is difficult to think of too many leap seconds, say 3 in a year. When $L$ approaches 20 , three leap seconds will probably have to be added in a year because of the perturbations of the periodic and irregular changes in the Earth's rotation. It will 
Table 1. The long-term trend of UTC vs. AT

\begin{tabular}{ccccccccccccccc}
\hline$E$ & $\Delta T$ & $L$ & $E$ & $\Delta T$ & $L$ & $E$ & $\Delta T$ & $L$ & $E$ & $\Delta T$ & $L$ & $E$ & $\Delta T$ & $L$ \\
\hline 10 & 00.3 & 0.3 & 110 & 036.3 & 06.3 & 210 & 132.2 & 12.3 & 310 & 288.3 & 18.3 & 410 & 504.3 & 24.3 \\
20 & 01.2 & 0.9 & 120 & 043.2 & 06.9 & 220 & 145.2 & 12.9 & 320 & 307.2 & 18.9 & 420 & 529.2 & 24.9 \\
30 & 02.7 & 1.5 & 130 & 050.7 & 07.5 & 230 & 158.7 & 13.5 & 330 & 326.7 & 19.5 & 430 & 554.7 & 25.5 \\
40 & 04.8 & 2.1 & 140 & 058.8 & 08.1 & 240 & 172.8 & 14.1 & 340 & 346.8 & 20.1 & 440 & 580.8 & 26.1 \\
50 & 07.5 & 2.7 & 150 & 067.5 & 08.7 & 250 & 187.5 & 14.7 & 350 & 367.5 & 20.7 & 450 & 607.5 & 26.7 \\
60 & 10.8 & 3.3 & 160 & 076.8 & 09.3 & 260 & 202.8 & 15.3 & 360 & 388.8 & 21.3 & 460 & 634.8 & 27.3 \\
70 & 14.7 & 3.9 & 170 & 086.7 & 09.9 & 270 & 218.7 & 15.9 & 370 & 410.7 & 21.9 & 470 & 662.7 & 27.9 \\
80 & 19.2 & 4.5 & 180 & 097.2 & 10.5 & 280 & 235.2 & 16.5 & 380 & 433.2 & 22.5 & 480 & 691.2 & 28.5 \\
90 & 24.3 & 5.1 & 190 & 108.3 & 11.1 & 290 & 252.3 & 17.1 & 390 & 456.3 & 23.1 & 490 & 720.3 & 29.1 \\
100 & 30.0 & 5.7 & 200 & 120.0 & 11.7 & 300 & 270.0 & 17.7 & 400 & 480.0 & 23.7 & 500 & 750.0 & 29.7 \\
\hline
\end{tabular}

be difficult to avoid changing the definition of the Atomic Second then, so that the Atomic Second could be close to UT second at that time. Alternatively, the limit on the difference would have to be increased.

At present, $L=7.8$ and the Atomic Second is shorter than UT second by $2.410^{-8}(=7.8 / 10 / 365.25 / 86400)$. If the definition of the atomic second were to be changed to 9192631997 (cf. Definition of AT above), the two seconds (AT and UT) would be equal, and there would have been a lot fewer leap seconds in the past 30 years. Of course, this conclusion is a measurement in hindsight and we can not be over critical to the predecessors. However the definition of Atomic Second apparently neglected the important fact: the UT second will be longer and longer. If we define an Atomic Second that is longer than the present UT second, UTC will have negative leap seconds first, then nearly zero or very few, and finally positive. Such a definition of an Atomic Second can be used for a much longer time span.

From Table 1 we see, that an $L=7.8$ in the past 27 years (average 1985) would correspond to $E=135$. So we have linked $E$ to the civil epoch: $E=150$ in $2000 \mathrm{AD}$. At $E=255$ (or AD 2070), $L$ would reach 15 and we shall have to think of a new, longer AT. If we choose a definition in which the Atomic Second exactly equals UT, $L$ will be less than 15 for 250 years. If we chose a longer AT, $L$ will be less than 15 for 500 years, etc.

Generally speaking, from Table 1 we can find how the long-term deceleration brings about leap seconds in UTC. By the means of a longer AT, a definition of the Atomic Second can fit a longer time span.

Because $E=135$ in $1985 \mathrm{AD}$, we get $E=0$ in $1850 \mathrm{AD}$. This is meaningful. The definition of the Atomic Second came from Ephemeris Time, i.e. the solar ephemeris. The ephemeris was based on observations made during the past 3 centuries where $1850 \mathrm{AD}$ is their weighted average epoch. Of course those observations were based on UT. In other words, the present Atomic Second was defined on the average UT second of $1850 \mathrm{AD}$. Therefore we have found another important fact, which was neglected when the Atomic Second was defined: ET second was the UT second at the 1850 mean epoch. The Atomic Second was too short at the very beginning because we adopted a definition of $1850 \mathrm{~s}$ in the 1970s! This

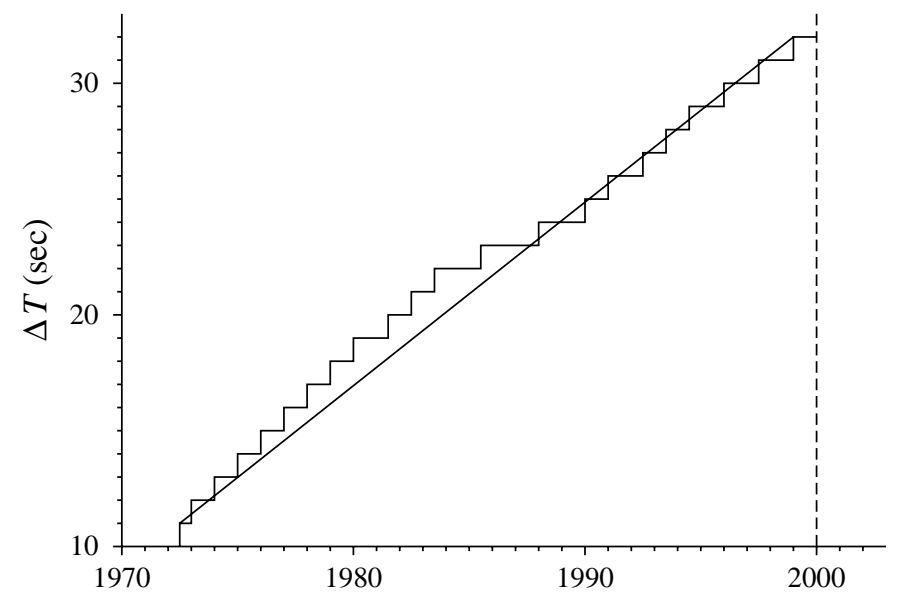

Fig. 1. The change of UTC vs. AT in the past years

is why the leap seconds have taken place frequently since the beginning, which was usually considered as a measurement error.

Of course it is not accurate to determine the epoch when the length of the UT second equals the Atomic Second only from the observations of past 30 years, although the problem has been shown clearly. Stephenson \& Morrison (1995) discussed this in detail. With observations during the past 300 years they got the epoch around $1800 \mathrm{AD}$, which is also the start epoch we used in the equation of this paper.

The Figure shows the leap seconds (step line) in UTC during the past 27 years, which gives an outline of UT1 vs. AT. If we had defined the atomic second as 9192631997 , it would show the oblique line and we would have had only 3 or 4 leap seconds during the past 30 years. If we had enlarged the limit of UTC from UT1 to 2 or $3 \mathrm{~s}$ (compared with 0.9 ), we would not have had any leap second at all during the 30 years!

Any way, leap seconds are a boring trouble in modern technology while precise time measurement does not need any relation with the Earth's rotation. On the other hand we have to indicate: if we simply eliminate leap seconds 
(to abandon UTC and adopt AT as civil standard time), it leads to increasing differences between standard time and UT1 - it will reach 1 hour in 11 centuries. In other words, it would be 13 o'clock rather than 12, at mean noon over the world, this would be a big impact to basic human concept! Therefore we have the following suggestion: by defining a new, properly longer Atomic Second and enlarging the limit of UTC from UT1, we shall not need any leap second in the next dozens of years.

Of course it is now difficult for us to change the definition of Atomic Second since so many basic physical constants are linked tightly to it. However the problem we pointed out in this letter would have been avoidable originally. This has offered a lesson that needs careful and comprehensive investigation when an important resolution is made.

\section{References}

Archinal, B. A. 1992, in Terrestrial coordinates and the rotation of the earth, Explanatory Supplement to the Astronomical almanac, ed. P. K. Seidelmann (University Science Books), 199

BIPM 1997, Annual Report of the BIPM Time Section, 10, 21

Dickey, J. O. 1995, Earth rotation variations from hours to centuries, in Highlights of Astronomy, 10, 17

Liu Ci-yuan 1994, Pubs. Shaanxi Obs., 17, 77

Pang, K. D., Yau, K., \& Chou, H. H. 1995, Pure and Applied Geophysics, 145, 459

Seidelmann, P. K., Guinot, B., \& Doggett, L. E. 1992, in Time, Explanatory Supplement to the Astronomical almanac, ed. P. K. Seidelmann (University Science Books), 39

Stephenson, F. R., \& Morrison, L. V. 1995, Phil. Trans. R. Soc. Lond., A351, 165 